\title{
Mesure d'éléments lourds émetteurs alpha, plus spécialement de l'uranium naturel, dans les liquides et sur extraits secs
}

\author{
A. ROBERT*
}

(Manuscrit reçu le 23 novembre 1993, révisé le 8 octobre 1994, accepté le 15 décembre 1994)

\begin{abstract}
RÉSUMÉ La méthode de détection alpha par scintillateur est appliquée à la mesure des sensibilités obtenues par des mesures sur un liquide, sur un dépôt de liquide, sur papier, sur un papier échangeur d'ions. Pour les éléments de numéro atomique élevé, les sensibilités de détection obtenues sont comparées par les techniques de détection alpha et de fluorescence $X$ non dispersives.
\end{abstract}

ABSTRACT The sensitivities of alpha detection using scintillator obtained on liquids, dry samples and ion exchange resin loaded paper were evaluated. For high atomic number elements, they were compared with results obtained by non-dispersive $x$-ray fluorescence methods and alpha-particle detection.

\section{Introduction}

La mesure directe de l'émission $\alpha$ d'un liquide trouve son intérêt à la fois pour des mesures en laboratoire et pour des mesures industrielles en ligne sur des volumes importants. La mesure de petites quantités en laboratoire permet de suivre et d'étudier un procédé, de déterminer le passage d'une élution à la sortie d'une colonne de chromatographie, par exemple. Les mesures industrielles s'adressent à la fois au contrôle de procédé (séparation des émetteurs $\alpha$ dans la dissolution de combustibles irradiés) et à la surveillance d'effluents radioactifs.

Les techniques de mesure $\alpha$ et en continu sur les liquides sont peu nombreuses. Une technique récente par scintillateur en contact direct avec le liquide à mesurer est utilisée ici. Les résultats obtenus avec cet appareillage, dans des conditions semblables mais avec divers radioisotopes, déterminent une limite de détection se situant de 2000 à $5000 \mathrm{~Bq} / 1[1,6,13-14]$. Nous avons tenté ici, en utilisant une technique de concentration d'émetteurs $\alpha$ en solution, d'estimer la nouvelle sensibilité que cet appareillage pouvait offrir. Les résultats obtenus sont comparés à ceux que présentent la fluorescence $\mathrm{X}$, soit sur le liquide, soit sur un échantillon solide.

* Laboratoire de physico-chimie des matériaux (LPCM), CNRS Bellevue, 92190 Meudon, France. 


\section{Le matériel de détection $\alpha$ utilisé}

L'appareillage DB 38 utilisé a été décrit par ailleurs [12,14]; il est constitué, pour l'essentiel, par un scintillateur de faible épaisseur [13] activé au cérium et mis en contact avec le liquide à mesurer. Le schéma de ce capteur est présenté figure 1. La cellule de mesure permet soit d'effectuer un passage continu du liquide, soit d'effectuer en séquentiel une mesure sur un échantillon de l'ordre de $1 \mathrm{ml}$. Une version munie d'un passeur d'échantillons permet également de mesurer des dépôts solides. L'originalité de cet appareillage est de ne pas interposer de fenêtre entre l'échantillon et le scintillateur. Celui-ci est insensible à la plupart des produits corrosifs et son diamètre utile de $30 \mathrm{~mm}$ présente une surface détectrice appréciable. Un rendement de détection de $84,5 \%$ a été mesuré à l'aide d'une source étalon de plutonium 239 [2].

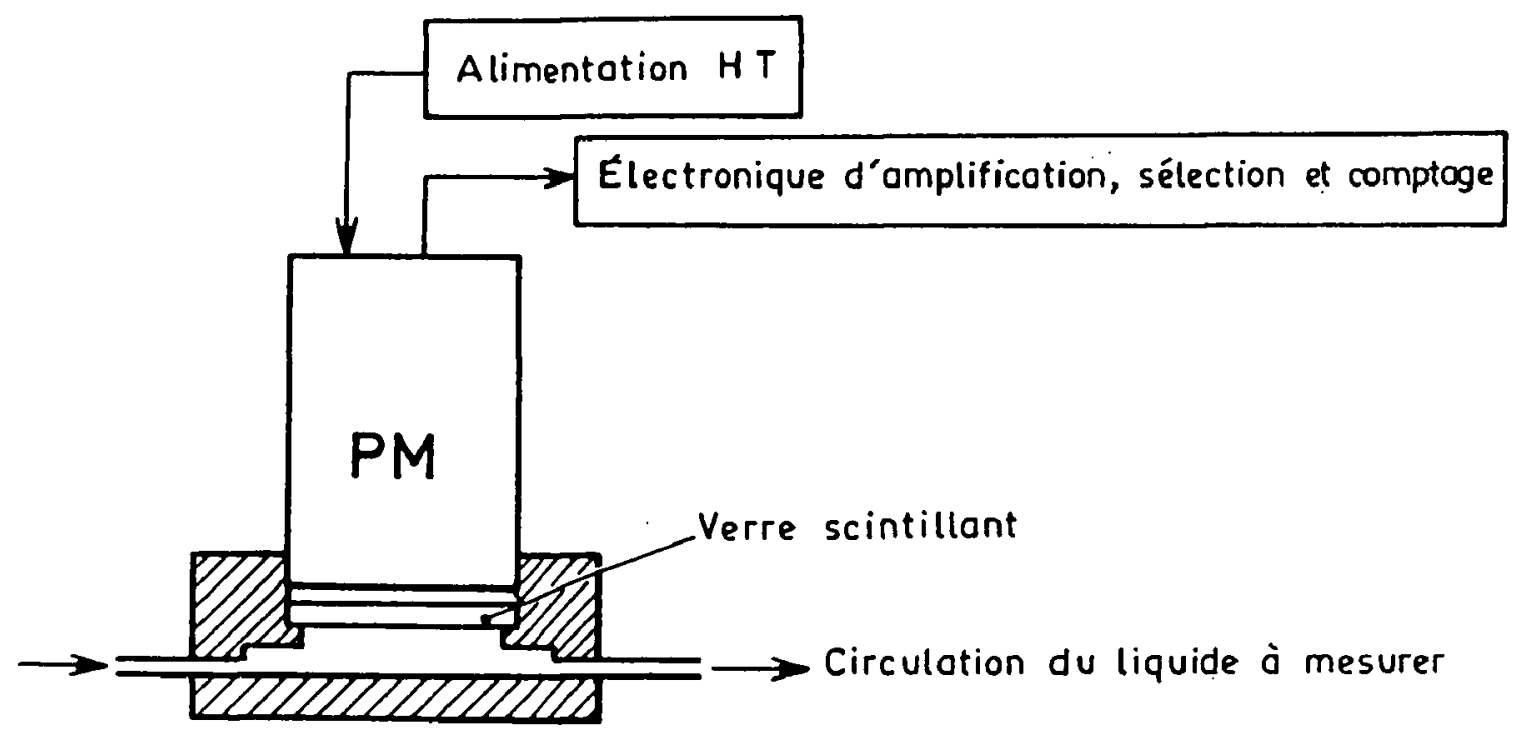

Fig. I - Schéma de l'appareil de mesure alpha. Diagram of the apparatus.

Le scintillateur utilisé pour les mesures présentées ici a une épaisseur de $15 \mu \mathrm{m}$, cette épaisseur correspondant à l'absorption complète du spectre $\alpha$ émergeant d'une solution. Les scintillateurs de faible épaisseur présentent un bruit de fond de faible valeur et sont donc particulièrement intéressants pour la détection des basses activités, l'expression de la limite de détection étant de la forme : $L D=f_{1}(B f)$. Le bruit de fond $(B f)$ d'un scintillateur est proportionnel à son volume, donc, ici, à son épaisseur $e$ si le diamètre est constant, soit donc : $L D=f_{2}(e)$.

Pour des diamètres constants, le bruit de fond a été mesuré en fonction de l'épaisseur du scintillateur. La figure 2 présente le résultat de ces mesures et confirme l'intérêt d'ajuster l'épaisseur du scintillateur au parcours des particules à détecter. Toutefois, si ces mesures de $B f$ ont bénéficié de conditions identiques, elles ne sont pas transposables à certaines mesures présentées plus 


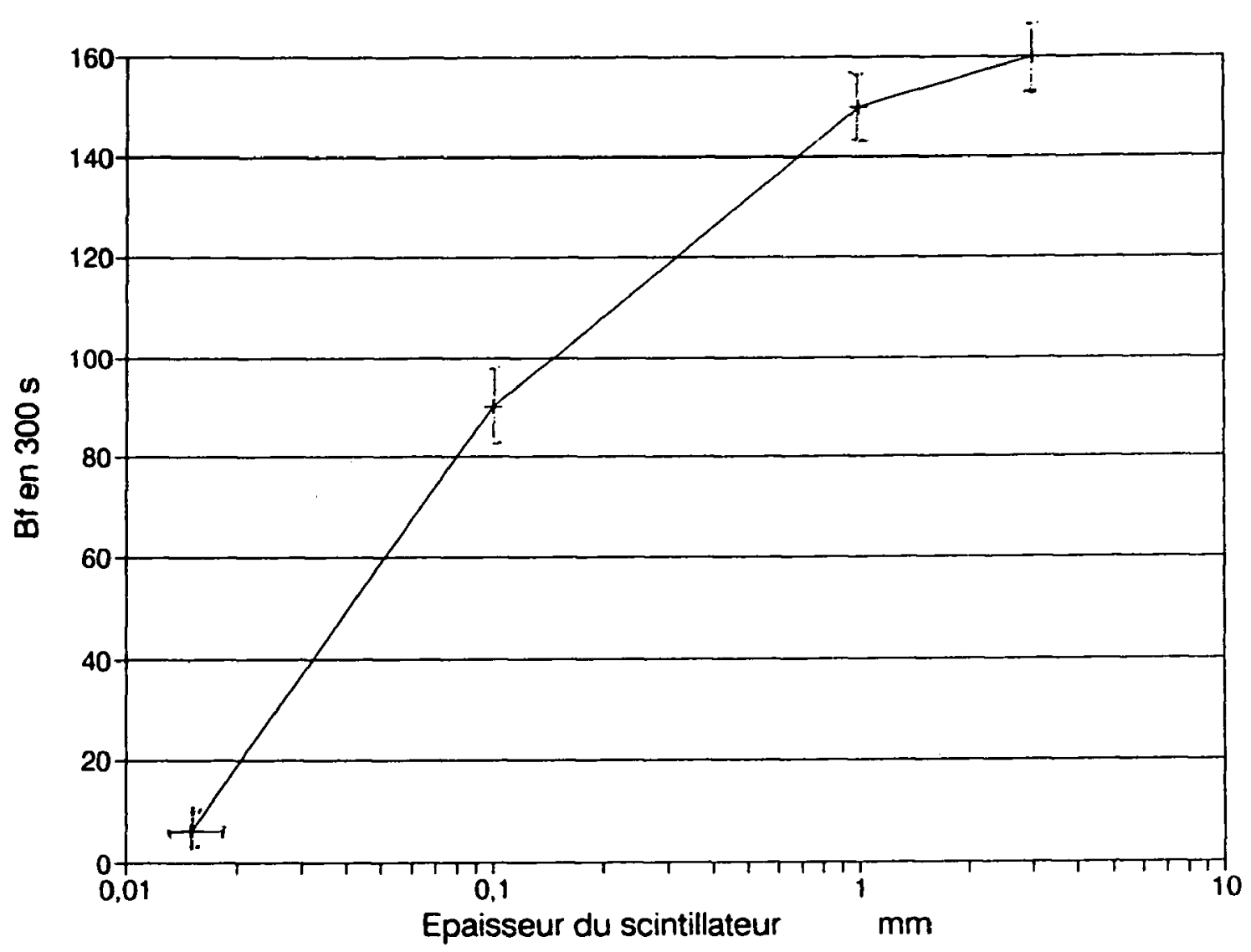

Fig. 2 - Bruit de fond.

Scintillator background vs thickness.

loin où une électronique différente donne une composante du bruit différente, où le rendement quantique des scintillateurs n'est pas le même et impose un seuil de discrimination différent.

Pour ces raisons, les comparaisons de sensibilité qui ont été obtenues suivant l'état de l'échantillon (liquide, dépôt sur papier ou sur échangeur d'ions) ne donneront ici qu'une tendance. La notion de limite de détection a servi de comparaison au détriment de la précision, mais a permis d'utiliser des séries d'échantillons ayant des teneurs volumiques très différentes. Pour atténuer l'incertitude due à des conditions de mesure qui n'ont pas été strictement identiques, deux séries de déterminations sont présentées, l'une sur l'uranium, l'autre sur l'américium 241 ou sur le plutonium 239.

\section{Mesures et résultats}

Les expressions utilisées pour définir le seuil de décision $S D$ et la limite de détection $L D$ se réfèrent aux travaux de Currie [5] et de la CETAMA [4]. Le seuil de décision est utilisé comme critère a posteriori pour décider si une grandeur a été détectée ou non. Il correspond à une mesure avec $100 \%$ d'incertitude aléatoire relative :

$$
S D=2(2 b / T)^{1 / 2}
$$

$b$ est le bruit de fond obtenu pendant le temps $T$ de la mesure. 


\section{A. ROBERT}

La limite de détection est employée pour qualifier la sensibilité d'une technique de mesure utilisant un appareillage déterminé et pour des conditions expérimentales données. $L D$ est calculé a priori et correspond à la plus faible activité que doit avoir l'échantillon à analyser pour pouvoir être déclaré détecté.

L'expression de la limite de détection est :

$$
\begin{aligned}
L D & =2 . S D \\
& =4(2 \mathrm{~b} / T)^{1 / 2}
\end{aligned}
$$

Les figures présentent des points de mesure auxquels se trouvent associées des barres d'erreur. Celles-ci correspondent à plus ou moins l'écart type et déterminent un intervalle de confiance de $68,3 \%$.

\subsection{Mesures directes sur liquide}

Les solutions d'uranium ont été préparées à partir de nitrate d'uranium Rhône-Poulenc dont le rapport ${ }^{235} \mathrm{U} / 238 \mathrm{U}$ n'est pas exactement connu mais n'est pas très différent de celui de l'uranium naturel. Les échantillons de solution d'uranium sont mesurés en $300 \mathrm{~s}$, et la figure 3 représente la réponse de l'appareil pour des concentrations de 0,5 à $10 \mathrm{~g} / \mathrm{l}$.

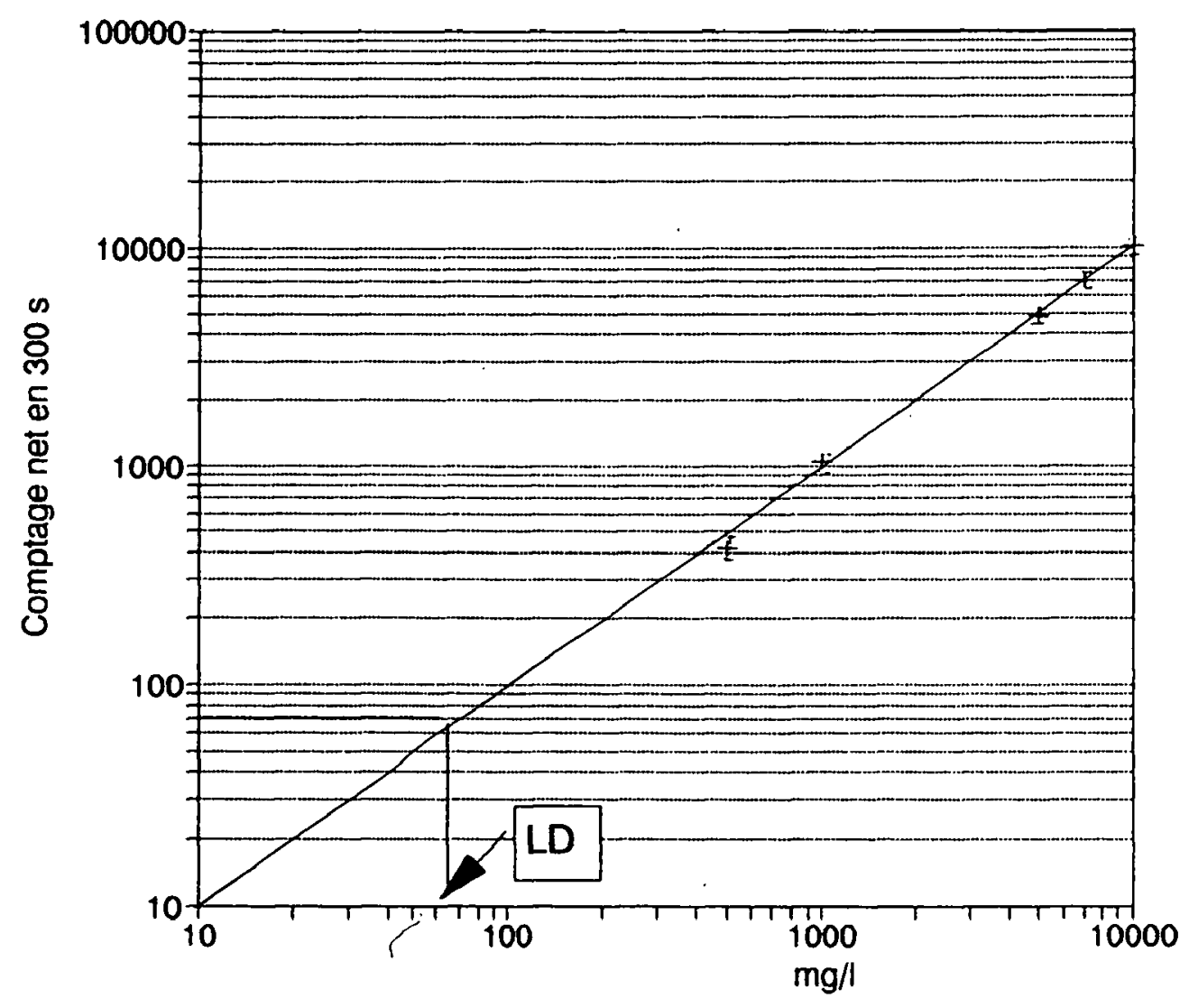

Fig. 3 - Uranium en solution.

Uranium determination, $1 N$ nitrate solution. 
On applique à ces résultats le calcul de la limite de détection précédemment défini, $T=300 \mathrm{~s}, b=160 / 300$.

Si $L D$ est exprimé pour un comptage de $300 \mathrm{~s}$, l'expression devient :

$$
\begin{aligned}
L D & =5,65(b / T)^{1 / 2} \times 300 \\
& =71,46 \text { impulsions en } 300 \mathrm{~s}
\end{aligned}
$$

L'ordonnée de $L D$ (Fig. 3) est 71,5 qui correspond, dans la limite des incertitudes, à une $L D$ en concentration de 60 à $70 \mathrm{mg} / \mathrm{l}$. Une valeur moyenne de $65 \mathrm{mg} / \mathrm{l}$ est portée dans le tableau I. Une valeur voisine $(50 \mathrm{mg} / \mathrm{l})$ de la $L D$ pour l'uranium est confirmée par Szabo [14] avec le même appareil DB 38 utilisé sur une boucle expérimentale du Service d'applications des radioéléments de Saclay. Les solutions utilisées pour cette mesure ont été préparées avec un uranium naturel certifié.

TABLEAU I

Limites de détection expérimentale

\begin{tabular}{|c|c|c|c|c|c|c|c|}
\hline Echantillon & $x^{2} x^{2}+2$ & liquide & Pats & dépo & papier & $\begin{array}{r}\text { dép } \\
\text { echang }\end{array}$ & $\begin{array}{l}\text { papier } \\
\text { Ir d'ions }\end{array}$ \\
\hline Radioélément & U nat & $241 \mathrm{Am}$ & 239Pu & Unat & Pinim & U Unat & ${ }^{239} \mathbf{P u}$ \\
\hline $\begin{array}{l}\text { mesure } \alpha \\
L D \mathrm{mg} / 1\end{array}$ & 50 & $35 \times 10^{-6}$ & $2 \times 10^{-3}$ & 620 & $214 \times 10^{-6}$ & 1,5 & $32 \times 10^{-6}$ \\
\hline $\begin{array}{l}\text { Coefficient } \\
\text { de corrélation }\end{array}$ & 0,9996 & 0,9980 & & 0,9928 & 0,9776 & 0,9943 & \\
\hline $\begin{array}{l}\text { Figure } \\
\text { correspondantes }\end{array}$ & 3 & 4 & & 5 & 6 & 8 & 8 \\
\hline $\begin{array}{l}\text { Fluorescence } \mathrm{X} \\
L D \mathrm{mg} / \mathrm{l}\end{array}$ & & 0,3 à 300 & & & & $10^{-3}$ à 0,1 & \\
\hline Références & & {$[9-10,14]$} & & & & {$[7,11]$} & \\
\hline
\end{tabular}
Experimental detection limits

La réponse de l'appareil à une solution nitrique d'américium 241 est représentée figure 4. L'expression de la limite de détection correspond à 15 impulsions. Cette intensité détermine sur la courbe une $L D$ en concentration de $35 \mathrm{ng} / \mathrm{l}$, elle correspond à une activité volumique de $4440 \mathrm{~Bq} / \mathrm{l}$. Pour faire une comparaison directe avec la technique de dépôt sur papier échangeur d'ions, il est calculé pour ${ }^{239} \mathrm{Pu}$ la concentration correspondant à la valeur de $4440 \mathrm{~Bq} / \mathrm{l}$. Ce calcul donne $1,956 \times 10^{-3} \mathrm{mg} / \mathrm{l}$; une valeur arrondie à $2 \times 10^{-3} \mathrm{mg} / \mathrm{l}$ est portée sur le tableau I, avec celle de $35 \times 10^{-6} \mathrm{mg} / 1$ pour ${ }^{241} \mathrm{Am}$. 


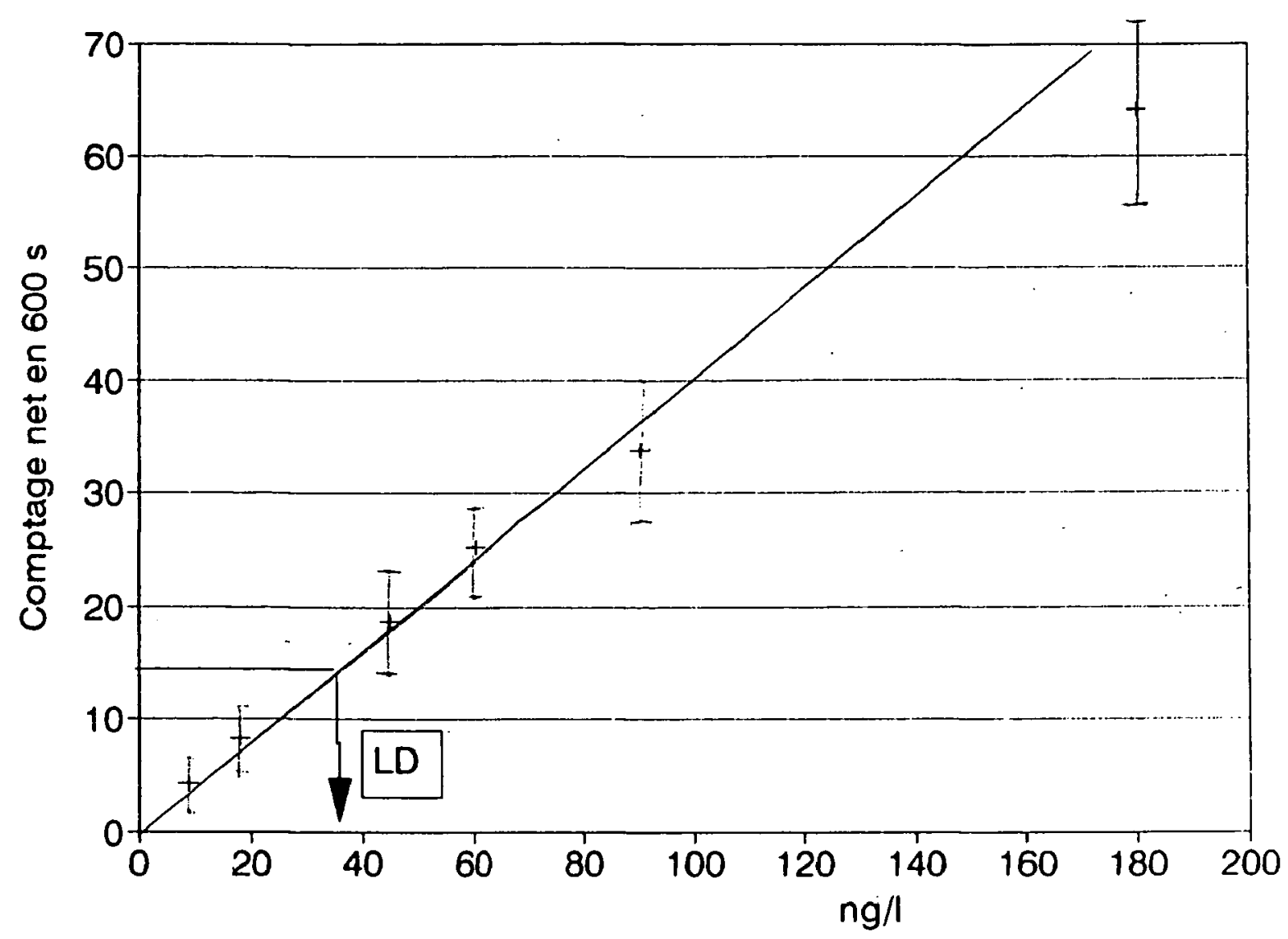

Fig. 4 - Américium 241 en solution.

Americium determination, nitrate solution.

Pour ce calcul, les données suivantes sont retenues :

$3,7 \times 10^{10} \mathrm{~Bq}$ correspond à $0,292 \mathrm{~g}$ de ${ }^{241} \mathrm{Am}$.

$2,27 \times 10^{9} \mathrm{~Bq}$ correspond à $1 \mathrm{~g}$ de ${ }^{239} \mathrm{Pu}$.

\subsection{Mesures de dépôts de solution sur papier}

Le but de la mesure de dépôts de solution sur papier est de préparer le dépôt sur papier échangeur d'ions et de se rendre compte de la variation de sensibilité apportée par cette technique vis-à-vis de la mesure directe sur solution.

Les solutions d'uranium sont déposées et évaporées sur un substrat de papier Joseph de $28 \mathrm{~mm}$ de diamètre. Le volume de solution déposé est de $0,1 \mathrm{ml}$ par échantillon. Le même appareillage de détection $\alpha$ est utilisé pour ces mesures à la différence, toutefois, que la cellule contenant le liquide est remplacée ici par un passeur d'échantillon.

Pour l'uranium, la figure 5 reproduit les mesures $\alpha$ effectuées sur des échantillons préparés avec des solutions nitriques et déposées sur papier. Pour un comptage de $300 \mathrm{~s}$, la limite de détection calculée est de $62 \mu \mathrm{g}$ d'uranium. Cette masse correspond à une solution de teneur volumique de $620 \mathrm{mg} / \mathrm{l}$. 


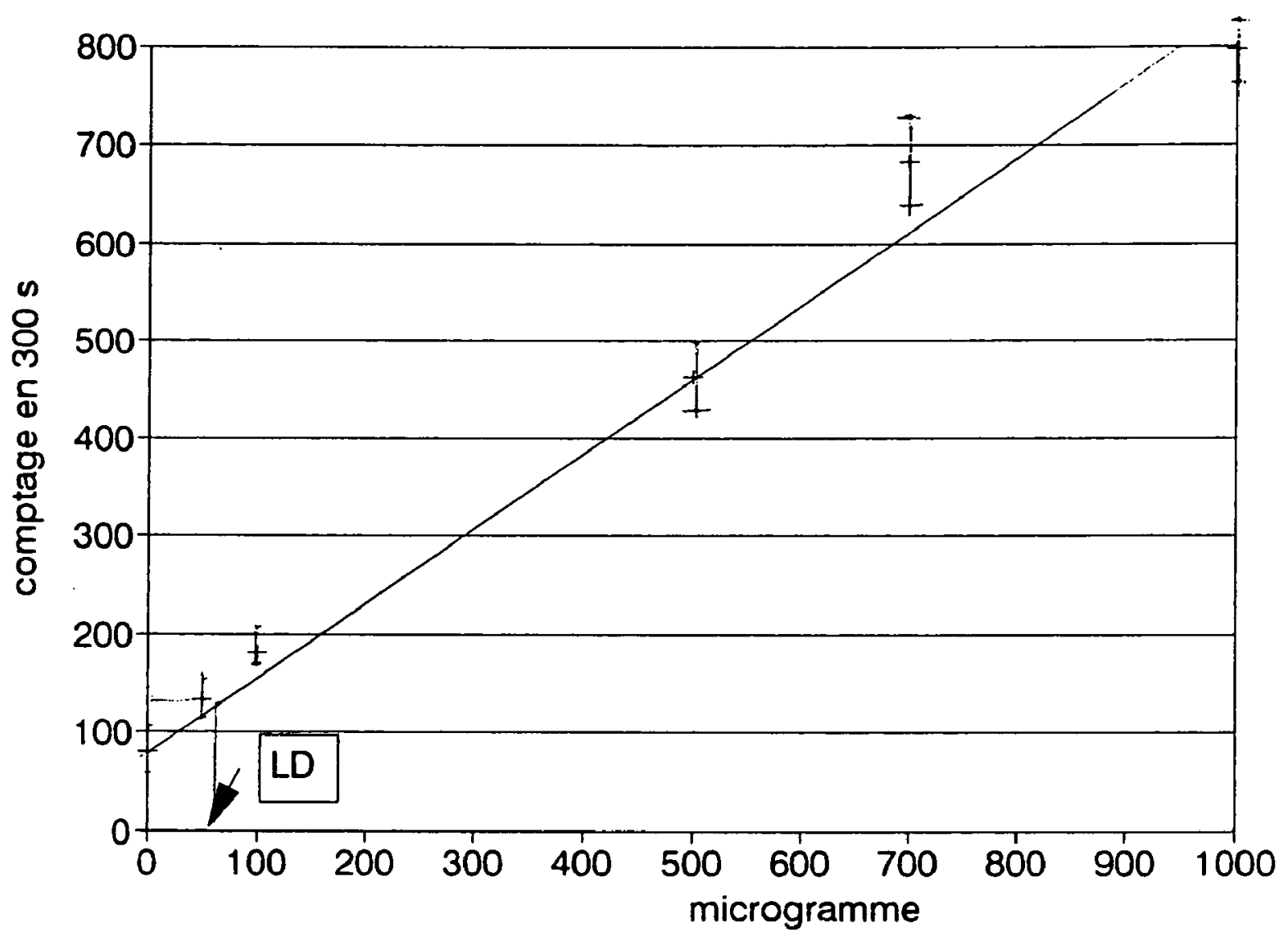

Fig. 5 - Uranium déposé sur papier.

Uranium solutions deposited on paper.

La même technique de préparation d'échantillon est appliquées à des solutions étalonnées d'américium 241. La figure 6 représente les mesures sur ces échantillons; on en déduit une $L D$ de 143 impulsions en $300 \mathrm{~s}$ pour le comptage. A ce taux de comptage correspond sur la courbe une masse évaluée entre 21,2 et $23,1 \mathrm{pg}$, la valeur de 21,4 pg est retenue ; elle correspond, pour un dépôt de $0,1 \mathrm{ml}$ sur le papier, à une concentration de $214 \mathrm{pg} / \mathrm{ml}$. Sur le tableau I, la valeur de $214 \times 10^{-6}$ est exprimée en $\mathrm{mg} / \mathrm{l}$. L'activité volumique associée est de $27,12 \mathrm{kBq} / 1$ de ${ }^{241} \mathrm{Am}$.

\subsection{Mesures sur papier échangeur d'ions}

L'intérêt porté aux résines échangeuses d'ions pour concentrer les éléments à l'état de traces n'est pas récent [15]; de nombreux travaux ont montré l'apport de cette méthode pour l'analyse des très faibles teneurs. La concentration de trace sur papier échangeur d'ions est devenue une méthode pratique de préparation d'échantillon.

Campbell et al. [3] ont examiné de façon approfondie les paramètres agissant sur l'échange ionique lors de la préparation d'échantillons sur papier échangeur d'ions. Cette technique de concentration et de préparation d'échantillons peut être appliquée à la mesure et au contrôle des émetteurs radioactifs 


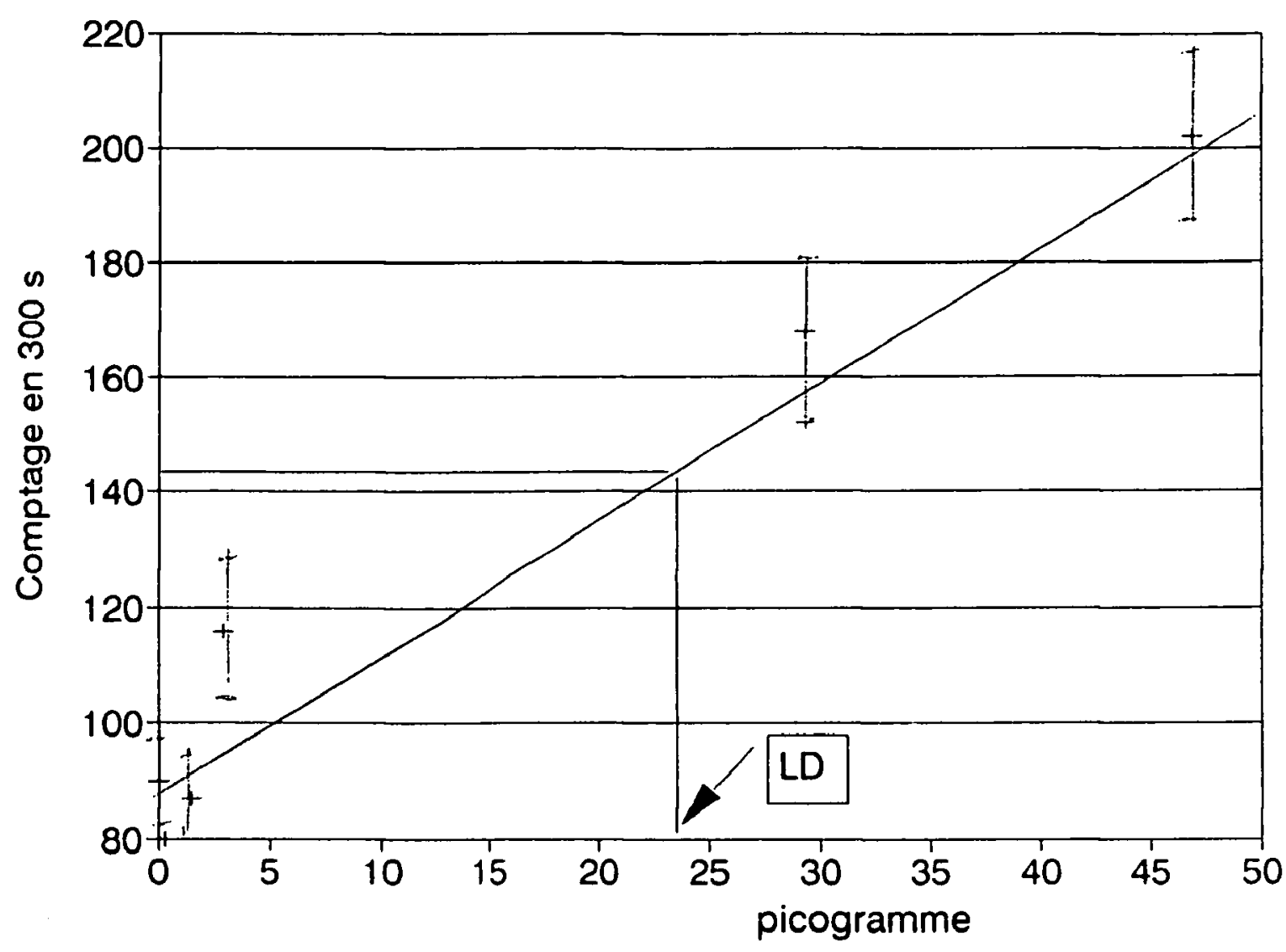

Fig. 6 - Américium 241 déposé sur papier.

Americium solutions deposited on paper.

contenus dans lẹ liquides; elle permet d'améliorer les sensibilités de détection à l'aide d'un appareillage simple et peu onéreux. Divers exemples d'applications de ce mode de préparation d'échantillons pour l'analyse ont été publiés pour des analyses de routine en fluorescence $X$. D. Joly [8] filtre sur un papier échangeurs d'ions la solution à analyser et annonce une limite de détection du plomb de $10^{-2} \mathrm{ppm}$.

En fluorescence $\mathrm{X}$ non dispersive et avec une excitation de l'échantillon par radioisotope, une limite de détection de $1 / 10 \mathrm{ppm}$ est obtenue sur les éléments lourds : $\mathrm{Ba}, \mathrm{Pb}$ et $\mathrm{U}$ [7]. Toujours en fluorescence $\mathrm{X}$, des limites de détection de l'ordre de la fraction de ppm ont été obtenues sur $\mathrm{Zn}, \mathrm{Pb}$ et $\mathrm{Cd}$ [8]. Dans ce dernier cas, la méthode de filtration de la solution à travers le papier échangeur d'ions a été remplacée par un simple trempage du substrat échangeur d'ions.

\section{a) Le papier échangeur d'ions}

Les papiers échangeurs d'ions utilisés sont constitués de cellulose très pure, imprégnée de façon homogène de $50 \%$ de leur masse par une résine échangeuse d'ions micropulvérisée. La concentration en résine par unité de surface est donc très élevée, procurant une grande efficacité d'échange. Pour notre étude, nous avons retenu les papiers SA-2 imprégnés de résine Amberlite 
IR120, convenant à la fixation des cations en milieu acide fort. L'épaisseur de ce papier est de $0,35 \mathrm{~mm}$. Les résines sont utilisées pour fixer quantitativement l'élément $\mathbf{M}$ à doser, qui se trouve sous forme ionique dans une solution très diluée. La fixation en milieu acide s'effectue suivant la réaction :

$$
\mathrm{H}_{\mathrm{R}}^{+}+\mathrm{M}^{+} \mathrm{S} \rightleftharpoons \mathrm{H}^{+} \mathrm{S}+\mathrm{M}^{+} \mathrm{R}
$$

Les indices $\mathbf{R}$ et $\mathrm{S}$ se rapportent aux ions dans la résine et aux ions dans la solution.

L'équilibre est caractérisé par un coefficient $K$ pour l'échange de l'ion $M^{+}$ entre la résine et la solution :

$$
\frac{\left[M^{+} \mathbf{R}\right]}{\left[M^{+} \mathrm{s}\right]}=K
$$

Ce coefficient dépend de la nature de $M$ et de celle de la solution; dans le cas de solutions très diluées, pour un $\mathrm{pH}$ convenable, $K$ est généralement très élevé, de telle sorte que la fixation de $M$ sur la résine est quantitative. Toutefois, si l'on se contente de plonger la résine dans la solution, l'équilibre peut être très long à atteindre ; aussi, pour accélérer les échanges, il est nécessaire de filtrer la solution à travers le papier.

\section{b) Régénération du papier}

Campbell indique que la régénération est nécessaire pour enlever $\mathrm{Ca}$ et les autres impuretés contenues dans la papier. De façon générale et aux teneurs où nous avons travaillé, cette régénération ne s'est pas révélée indispensable. Nous considérons donc la régénération comme une précaution; elle peut aussi être effectuée pour une réutilisation de papier ayant déjà servi.

Pour régénérer on procède comme suit : après un trempage du papier de quelques minutes dans de l'eau distillée, on filtre 5 fois $40 \mathrm{~cm}^{3}$ d'acide chlorhydrique $6 \mathrm{~N}$; la résine est alors sous forme ionique $\mathrm{H}^{+}$. Ensuite, on filtre 6 fois $40 \mathrm{~cm}^{3}$ d'une solution saturée de chlorure de sodium pour redonner à la résine sa forme ionique $\mathrm{Na}^{+}$et, finalement, on lave à l'eau bidistillée afin d'éliminer l'excès de chlorure de sodium. Pour contrôler que le lavage est terminé, on ajoute quelques gouttes d'une solution de sels d'argent ou de plomb dans le filtrat ; aucun trouble ne doit apparaître.

\section{c) Préparation de l'échantillon}

Le papier est utilisé sous forme de disque de $30 \mathrm{~mm}$ de diamètre, découpé à l'emporte-pièce. Le disque est préalablement trempé $15 \mathrm{~min}$ dans de l'eau distillée. On définit à $40 \mathrm{~cm}^{3}$ le volume de la solution à concentrer. Ce volume est amené au pH 2 par addition de $\mathrm{HNO}_{3}$ concentré. Une mesure au papier $\mathrm{pH}$ est jugée suffisante. Le disque après trempage est placé dans un appareil de filtration constitué d'un entonnoir à fond de verre fritté sur lequel le substrat échangeur d'ions repose (Fig. 7). L'appareil est placé sur un Büchner et mis 


\section{A. ROBERT}

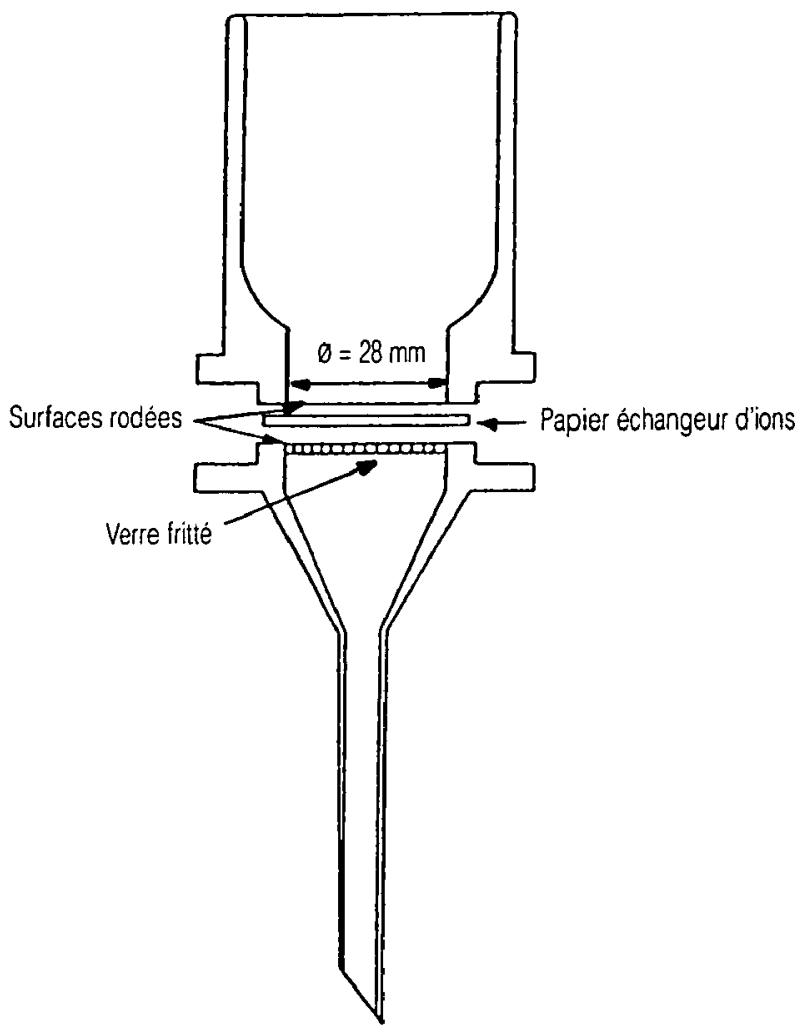

sous vide par une trompe à eau, de telle sorte que l'on régule la vitesse de filtration à l'aide d'une fuite réglable. Au-delà de 10 à $20 \mathrm{~s}$, le temps de passage du liquide sur le papier échangeur d'ions n'influe pas sur le taux d'échange $[3,11]$. Par contre, il est indispensable que le liquide passe plusieurs fois sur le papier ; des essais nous ont montré que 7 passages assurent l'équilibre ou la saturation. Avant ces opérations, les faces du disque ont été repérées afin de présenter à la mesure celle qui reçoit le liquide pendant la filtration. La circulation du liquide terminée, le disque est retiré et séché entre deux feuilles de papier Joseph.

Fig. 7 - Appareil à filtrer en verre. Filtration apparatus.

Remarque : si l'élément à déterminer se trouve en très faible teneur en présence de constituants majeurs pouvant être fixés, il conviendra alors de séparer l'élément à mesurer pour que le papier échangeur d'ions ne se trouve pas saturé.

\section{d) Mesures $\alpha$}

Les conditions de la mesure $\alpha$ sont identiques à celles décrites précédemment pour le cas des préparations par évaporation. Pour évaluer l'amélioration de la sensibilité résultant de cette préparation d'échantillon, 2 types de solutions sont employées. Des solutions d'uranium naturel de diverses concentrations et une solution où ${ }^{239} \mathrm{Pu}$ se trouve à l'état de trace.

Pour l'uranium naturel, une solution mère $\mathrm{UO}_{2}\left(\mathrm{NO}_{3}\right)_{2}$ est préparée, puis diluée pour donner les concentrations en U s'étalant de 10 à 0,2 ppm. La figure 8 présente le comptage en $300 \mathrm{~s}$ en fonction de la teneur en ppm des solutions filtrées súr papier SA.2. La limite de détection de $1,5 \mathrm{ppm}$ ou $1,5 \mathrm{mg} / \mathrm{l}$ est indiquée par une flèche sur l'axe des abscisses de la figure 8.

Des traces de ${ }^{239} \mathrm{Pu}$ ont été filtrées sur cette même qualité de papier échangeur d'ions. La solution $\mathrm{HNO}_{3}, 1 \mathrm{~N}$ de volume $40 \mathrm{ml}$, contient le plutonium, son activité volumique est de $80 \mathrm{~Bq} / \mathrm{l}$ plus ou moins $10 \%$. L'échantillon obtenu est mesuré dans les mêmes conditions que celles de l'uranium et le résultat est 


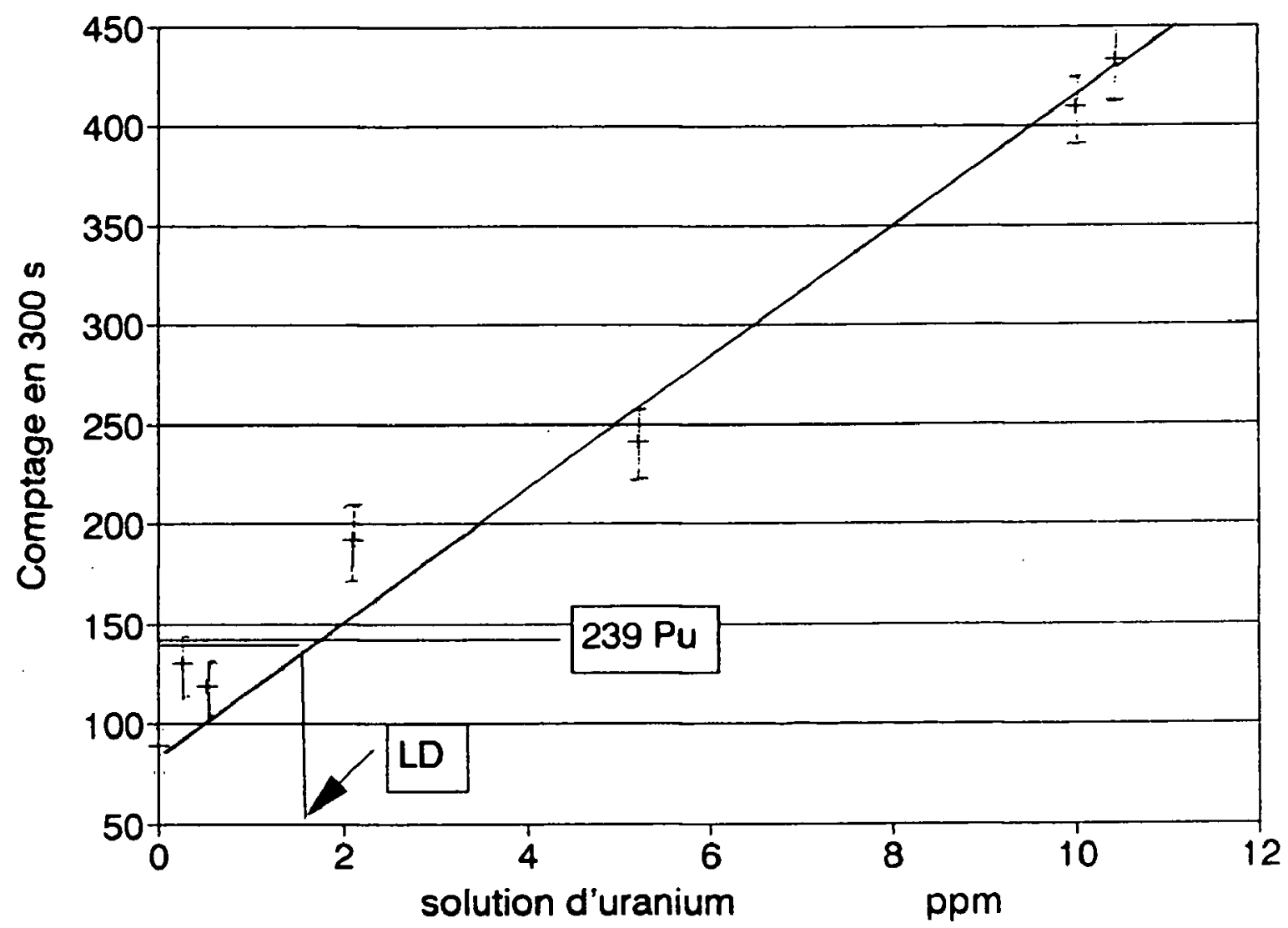

Fig. 8 - Comptage sur échangeur d'ions.

$U$ and $P u$ on ion exchange resin loaded paper.

porté sur la figure 8 . La valeur de 146 impulsions en $300 \mathrm{~s}$ se trouve légèrement au-dessus de la limite de détection déterminée pour les échantillons d'uranium. Pour le plutonium 239, on en déduit une limite de détection de 75 à $80 \mathrm{~Bq} / \mathrm{l}$.

\section{Discussion et conclusions}

Le tableau I permet de comparer les sensibilités obtenues pour les trois conditions de mesure présentées ici. L'amélioration de sensibilité apportée par une préparation de l'échantillon sur papier échangeur d'ions est manifeste. Si cette dernière méthode ne permet pas la réponse instantanée d'une mesure directe sur le liquide $(20 \mathrm{~s}$ ), elle reste relativement rapide (environ $10 \mathrm{~min}$ ) et n'exige pas de matériel onéreux. Malgré la dispersion apportée par les préparations d'échantillons et l'erreur inhérente à l'expression de la limite de détection, les résultats présentés par les couples de mesures uranium et américium ou plutonium sont homogènes.

La méthode accessoire de dépôt de solution sur papier présente des coefficients de corrélation les plus faibles du tableau. De plus, la $L D$ pour ces mesures est supérieure à celle que présentent les mesures sur solution. La dispersion et la perte de sensibilité sont liées au mode de préparation de l'échan- 


\section{A. ROBERT}

tillon et à la nature du substrat recevant la solution. Ici, un volume important de solution est dispersé dans un matériau inhomogène. Le réseau fibreux du papier absorbe la solution en profondeur et crée ainsi l'atténuation de l'émission apha.

Par cette observation, on retrouve l'intérêt bien connu d'effectuer des dépôts sur des surfaces métalliques polies. Dans le cas présent, le seul intérêt de ces dépôts sur papier a été celui de préparer les conditions de dépôt sur papier échangeur d'ions. Ces papiers chargés de résine et le mode de préparation (circulation de la solution) donnent (Tab. I) des coefficients de corrélation acceptables se rapprochant de ceux observés pour les mesures sur solution. Il est aussi à remarquer que ces dépôts sur échangeur d'ions sont stables, des reprises de mesures sur plusieurs années montrent la possibilité de les utiliser comme référence sur une longue période.

Ce même tableau I présente quelques résultats publiés illustrant les performances obtenues par la technique de fluorescence $X$, pour des mesures sur solution et aussi sur papiers échangeurs d'ions. Dans ce dernier cas, des travaux de laboratoire [7-8] revendiquent pour l'uranium des résultats meilleurs que ceux obtenus par comptage alpha. On retrouve une meilleure sensibilité sur l'uranium par les techniques industrielles de fluorescence $X$ sur solution [9-10]. Malgré un appareillage toujours plus onéreux que celui utilisé pour les mesures alpha, Szabo [14] montre que la fluorescence X présente, en dehors de l'uranium, des $L D$ bien inférieures à celles obtenues par comptage alpha.

\section{Remerciements}

Les facilités accordées par M. Blanzat, directeur du LPCM au CNRS m'ont permis d'entreprendre ce travail. Que M. le Pr Blanc trouve ici l'expression de ma gratitude pour l'intérêt qu'il a porté à mes travaux. Le matériel de mesure a été prêté par la Société SA2 I.

\section{RÉFÉRENCES}

[1] Appareil de mesure des particules $\alpha$ en solution MAS 38 - Rapport d'essais CTHIR 93-092, 1993.

[2] BERES A., BIANCHI C., LECROART B. - Qualification d'un détecteur de particules alpha (DB38). Note technique CEA-DRDD-SAP-NT 93-01, 1993.

[3] CAMPBELL W.J., SPANO E.F., GREEN T.E. - Micro- and trace analysis by a combination of ion exchange resin-loaded papers and $\mathrm{x}$-ray spectrography. Anal. Chem., 1966, 38 (8) 987-996.

[4] COMMISSION D'ETABLISSEMENT DES METHODES D'ANALYSES (CETAMA) - Groupe de travail $\mathrm{n}^{\circ} 11$. Limites de détection. Rapport CETAMA, 1990.

[5] CURRIE L.A. - Limits for qualitative detection and quantitative determination, application to radiochemistry. Anal. Chem., 1968, 40 (3) 586-593. 
[6] HEINDL R., SELLA C., ROBERT A. - RF sputtered Ce+3 activated $\mathrm{SiO}_{2}$ glass films as scintillators for alpha particles detections. 5. International conference on ion and plasma assisted technics IPAT 85, Munich, 1985.

[7] HOLYNSKA B., LESZKO M., NAHLIK E. - The application for ion-exchanger foils in the determination of trace amounts of some metals in water by means of the non-dispersive $\mathrm{X}$-ray fluorescence method. J. Radional. Chem., 1973, 13, 401-411.

[8] JOLY D. - Dosage de très faible traces en solution par utilisation de papiers échangeurs d'ions. In : 8. Colloque Philips sur l'analyse de la matière, Florence, 1969, 7584.

[9] MARTINELLI P. - Determination of $U$ an/or Pu using x-ray fluorescence analysis excited by ${ }^{192}$ Ir sealed sources. Nucl. Instrum. Meth., 1985, $A$ 242, 569-574.

[10] ROBERT A. Gerät zur Röntgenemissionanalyse mittels Nachweises niederenergetischer Strahlung bei Anregung durch eine Radionuklidstrahlungsquelle. Isotopenpraxis, 1973, 5 (9), 153-159.

[11] ROBERT A., VALLES R. - Analyses de traces par concentration sur papier échangeur d'ions et fluorescence $X$ radioisotopique. Radiochem. Radioanal. Lett., 1973, 15 (4-5), 279-289.

[12] ROBERT A., SELLA C., HEINDL R. - Measurement system for $\alpha$ emitters in solution. Nucl. Instrum. Methods, 1984, 225 (1), 179-184.

[13] ROBERT A. - Détection de particules $\alpha$ par un scintillateur constitué d'un film de $\mathrm{SiO}_{2}$ activé par $\mathrm{Ce}{ }^{+3}$ et obtenu par pulvérisation radiofréquence. CEA - Conf -8225 , 1985 .

[14] SZABO J.L., LAMARQUE G., JUNCA R., FROMENTIN A. - Comparaison de la limite de détection de l'uranium en solution de quelques appareillages d'analyse. Caractérisation et bilan financier. Note technique CEA-NT-DAMRI-SAR-S 93-435, 1993.

[15] Van NIEKERK J.N., De WET J.F. - Trace analysis by x-ray fluorescence using ion exchange resins. Nature, 1960, 186, 380-383. 